ఠ

\title{
Modeling the alternative oxidase from the human pathogen Blastocystis using automated hybrid structural template assembly
}

This article was published in the following Dove Press journal:

Research and Reports in Biochemistry

II January 2012

Number of times this article has been viewed

\author{
Daron M Standley' \\ Mark van der Giezen² \\ 'Laboratory of Systems Immunology, \\ World Premier International \\ Immunology Frontier Research \\ Center, Osaka University, Osaka, \\ Japan; ${ }^{2}$ Centre for Eukaryotic \\ Evolutionary Microbiology, \\ Biosciences, College of Life and \\ Environmental Sciences, University \\ of Exeter, Exeter, UK
}

\begin{abstract}
Alternative oxidases ( $\mathrm{AOX}$ ) of human parasites represent attractive drug targets due to their absence in humans. However, the lack of a structure has prevented structure-based drug design. Moreover, a large helical insertion proves difficult for automated structural modeling efforts. We have used a novel hybrid structural modeling approach to generate a model that is globally consistent with a previous model but based on a phylogenetically closer template and systematic sampling of known fragments in the helical insertion. Our model, in agreement with site-directed mutagenesis studies, clearly assigns E200 as the iron-ligating residue as opposed to the previously suggested E201. Crystallization of AOX from another species has recently been reported suggesting that our blind prediction can be independently validated in the near future.
\end{abstract}

Keywords: homology modeling, protein structure, blind prediction, fragment assembly, active site, parasite, mitosome, hydrogenosome, evolution

\section{Introduction}

Blastocystis is a widely distributed human pathogen of uncertain pathogenicity. ${ }^{1}$ Approximately $10 \%$ of the population in developed countries carries the parasite while figures as high as $60 \%$ have been reported in developing nations. ${ }^{2}$ Blastocystis is the only human infectious agent of the stramenopiles (if one ignores the very rarely pathogenic oomycete Pythium), a taxon grouping containing more than 100,000 species. Understanding the unusual metabolism of a human parasite from an evolutionary distant group might reveal potential therapeutic targets, such as the alternative oxidase (AOX), which is absent in humans. The AOX of Trypanosoma brucei, the causative agent of sleeping sickness, has been studied for exactly this reason. ${ }^{3,4}$ AOXs belong to the di-iron carboxylate family and function as non-proton-pumping quinol oxidases that short-circuit the normal mitochondrial electron transport chain, because they do not allow the feed-through of electrons to Complex III. AOX transfers electrons directly from the quinol pool (from Complex II) to oxygen and therefore does not contribute to the proton gradient used by the $\mathrm{F}_{0} \mathrm{~F}_{1}$ ATP synthase to generate ATP. Interestingly, an AOX was recently discovered in Blastocystis. ${ }^{5}$ Although considered wasteful in aerobic mitochondrial metabolism, for anaerobes such as Blastocystis without downstream electron transport complexes, AOX might perhaps be the terminal electron acceptor. No genuine alternative acceptor has thus far been identified in its genome. ${ }^{6}$

Progress in understanding the mechanism of AOX has been hampered by the lack of a solved crystal structure. A current search of structural templates using the BioInfoBank MetaServer ${ }^{7}$ consistently places AOX within the Ferritin-like SCOP
Correspondence: Mark van der Giezen Centre for Eukaryotic Evolutionary Microbiology, Biosciences, College of Life and Environmental Sciences, University of Exeter, Stocker Road, Exeter EX4 4QD, UK

Tel +44 I 392723483

Fax +44 I392 723434

Email m.vandergiezen@exeter.ac.uk
Research and Reports in Biochemistry 2012:2 I-8

(C) 2012 Standley and van der Giezen, publisher and licensee Dove Medical Press Ltd. This is an Open

Access article which permits unrestricted noncommercial use, provided the original work is properly cited.
Dovepress

http://dx.doi.org// 0.2147/RRBC.S26820 
(structural classification of proteins) superfamily, which consists of a diverse group of mostly helical bimetal-binding proteins. According to the SCOP database, the Ferritin-like superfamily now consists of nine different families, the most populated being Ferritin itself, with 34 members, and Ribonucleotide reductase-like, with 18 members. Several structural models have been put forward to explain the function of AOX, the most recent being the 1999 Andersson and Nordlund model ${ }^{8}$ which used $\Delta^{9}$-desaturase, a member of the Ribonucleotide reductase-like family, as a structural template. This model was consistent with conserved residues as well as predicted secondary structure. The choice of $\Delta^{9}$-desaturase as a template was attributed primarily to the spacing of the iron-coordinating residues. However, profileprofile sequence alignment indicates a more statistically significant similarity to members of the Ferritin-like family, such as Tm1526 from Thermotoga maritima, a functionally uncharacterized structural genomics target. Structures such as Tm1526 represent an opportunity for improving predictions of the AOX structure, and thus enhance our chances for structure-based design of an inhibitor.

One of the challenges of modeling AOX on structurally characterized members of the Ferritin-like superfamily is that AOX is predicted to contain a $\sim 28$ residue helical region that is not present in the current repertoire of structural templates. Consequently, if standard structural modeling techniques are used, this region must either be ignored, modeled carefully by hand, or treated as a random coil. Because the region in question is predicted to anchor AOX to the mitochondrial inner membrane, where it can interact with quinones, ${ }^{8}$ and is therefore physiologically relevant, it is important to model the entire region as accurately as possible.

One of us recently introduced a fragment assembly method Spanner, ${ }^{9}$ which uses hybrid templates to generate a gap-free alignment to the query. The choice of template fragments includes both primary and secondary structural information from the "query" (AOX) as well as tertiary structural information from the main template (Tm1526). The Spanner model of AOX, using state-of-the art profile-profile alignment, agrees in most respects with the earlier model, but an important difference is that the model clearly places E200 in an iron-coordinating position, rather than E201, as predicted previously. ${ }^{8}$ Because crystallization of AOX from T. bruce $i$ has recently been reported, ${ }^{10}$ an X-ray structure is expected to be released in the near future. This gives us an opportunity to make a blind prediction of the structure of $\mathrm{AOX}$, in particular with respect to coordination of the iron atoms and helical insertion.

\section{Results}

\section{Sequence to structure alignment}

The sequence of Blastocystis AOX was first submitted to the BioInfoBank MetaServer ${ }^{7}$ in order to obtain the consensus sequence window (N102-A304) aligning to the Ferritin-like SCOP superfamily. We then utilized the FFAS03 profileprofile alignment server ${ }^{11}$ to select the closest known SCOP domain for this region. As discussed above, a model was previously constructed using $\Delta^{9}$-desaturase, a member of the Ribonucleotide reductase-like family (SCOP identifier a.25.1.2) as a template. ${ }^{8}$ In Table 1 we show the FFAS03 scores to the top 10 SCOP hits. It is clear from this table that the magnitude of the scores to two uncharacterized members of the Ferritinlike family (SCOP identifier a.25.1.1, with scores -14.10 and -13.10 , respectively), are higher than that of the highestscoring member of the Ribonucleotide reductase-like family (M. tuberculosis Desa2 with a score of -8.79). These results are a reflection of the improvement in structural coverage from structural genomics efforts: the three highest-scoring hits, which lie well above the default FFAS03 score threshold of nine, are structural genomics targets that were not available at

Table I Top FFAS SCOP hits

\begin{tabular}{|c|c|c|c|c|c|}
\hline Rank & Score & PDB & Family & Name & Species \\
\hline I & -14.10 & Ivjx & a.25.l.I & Hypothetical protein TmI526 & Thermotoga maritima \\
\hline 2 & -13.10 & $2 f z f$ & a.25.l.l & Hypothetical protein PfI I 90 & Pyrococcus furiosus \\
\hline 3 & -11.30 & $20 h 3$ & a.25.1.8 & Uncharacterized protein & Magnetospirillum magnetotacticum \\
\hline 4 & -9.82 & Ijkv & a.25.1.3 & Manganese catalase & Lactobacillus plantarum \\
\hline 5 & -8.79 & Iyuz & a.25.l.I & Nigerythrin & Desulfovibrio vulgaris \\
\hline 6 & -8.79 & $\mathrm{IzaO}$ & a.25.1.2 & Desa2 & Mycobacterium tuberculosis \\
\hline 7 & -8.59 & Inf4 & a.25.l.l & Bacterioferritin & Desulfovibrio desulfuricans \\
\hline 8 & -8.54 & Ilko & a.25.I.I & Rubrerythrin & Desulfovibrio vulgaris \\
\hline 9 & -8.29 & $1 j 30$ & a.25.l.I & Hypothetical rubrerythrin & Sulfolobus tokodaii \\
\hline 10 & -8.04 & $2 \mathrm{cwl}$ & a.25.1.3 & Manganese catalase & Thermus thermophilus \\
\hline
\end{tabular}

Notes: The top-10 hits to SCOP domains are shown. The "Family" column lists the SCOP family classification. The top 4 hits score above the default FFAS confidence threshold. Family a.25.I.I is the Ferritin-like family while a.25.I.2 is the Ribonucleotide reductase-like family from which the previous template was taken. ${ }^{8}$ Abbreviation: SCOP, structural classification of proteins. 
the time the $\Delta^{9}$-desaturase model was built. The fourth-highest scoring template, which is not a structural genomics target, was also unavailable at the time.

\section{Phylogenetic analysis}

Although phylogenetic analyses of the Ferritin-like superfamily have been reported, ${ }^{12,13}$ the distances within this extensive protein family are too large to enable meaningful protein substitution model-enabled analysis. We therefore decided to compare the relationships between a smaller subset of sequences from the following subfamilies: alternative oxidase, $\Delta^{9}$-desaturase, ferritin-like, and ferritin. Both our Bayesian as well as our maximum likelihood analysis clearly clustered the alternative oxidases away from the $\Delta^{9}$-desaturases with strong bootstrap and posterior probability support (Figure 1) and these two subfamilies were separated by the ferritin-like and ferritin subfamilies. The nodes leading to the ferritinlike sequences received no significant support and appear to cluster intermediately between $\Delta^{9}$-desaturases and alternative oxidases. Although phylogenetic and structural similarity are not synonymous (see the striking example of citrate synthase $)^{14}$ our analysis provides support for the notion that other sequences than $\Delta^{9}$-desaturase might provide better templates to model alternative oxidases.

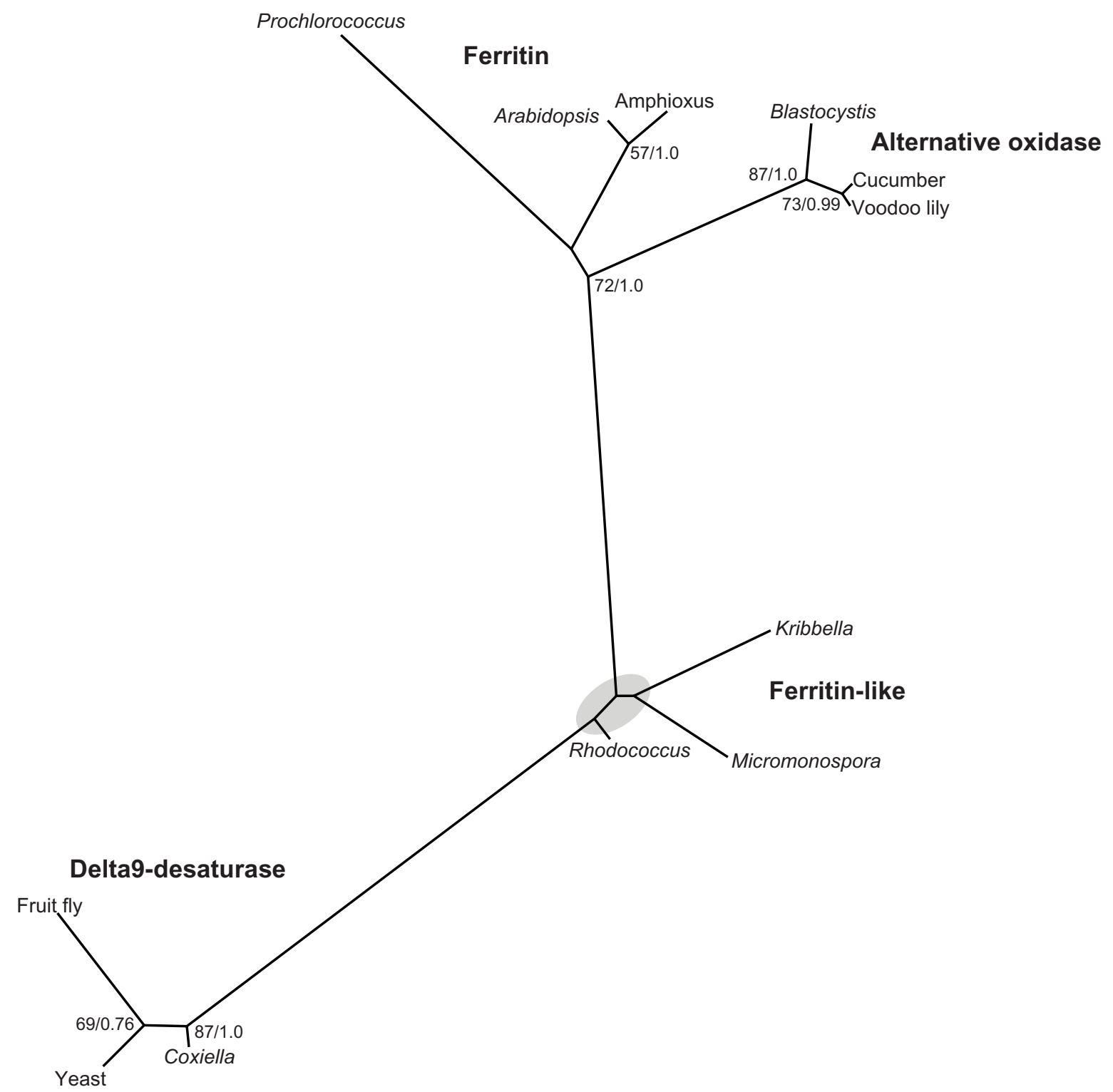

Figure I Phylogenetic analysis of alternative oxidases, ferritins, ferritin-likes and $\Delta^{9}$-desaturases. An unrooted maximum likelihood phylogenetic tree of I 2 members of the large ferritin-like superfamily is shown. The alternative oxidases are recovered as part of a well supported monophyletic group distant from the $\Delta^{9}$-desaturases. Numbers at the nodes represent bootstrap values as determined using $\mathrm{PHYML}^{24}$ (only bootstrap values above $50 \%$ are shown) and posterior probabilities as determined by MrBayes ${ }^{23}$ where a value of 1.0 represents maximum support (only values above 0.75 are shown). The gray zone indicates nodes that were unresolved in the Bayesian analysis. 


\section{Structural modeling}

A structural model of Blastocystis AOX was constructed from alignment to the highest-scoring template, Tm1526 from T. maritima, as shown in Figure 2. Although the sequence identity to the template was only $11 \%$, the iron-coordinating residues were well conserved, as discussed below. As mentioned in the Introduction, in order to obtain a model that matched the predicted secondary structure, a hybrid template modeling approach was used. AOX contains a predicted helix in the region A164-S192 that is not present in the Ferritinlike SCOP superfamily. We thus edited the alignment in this region so that the predicted helical region corresponded to a large gap. The resulting alignment (Figure 2A) was then submitted to the Spanner server (http://sysimm.ifrec.osaka-u. ac.jp/spanner/). Spanner uses structural fragments to generate a hybrid template such that there are no gaps with the query.
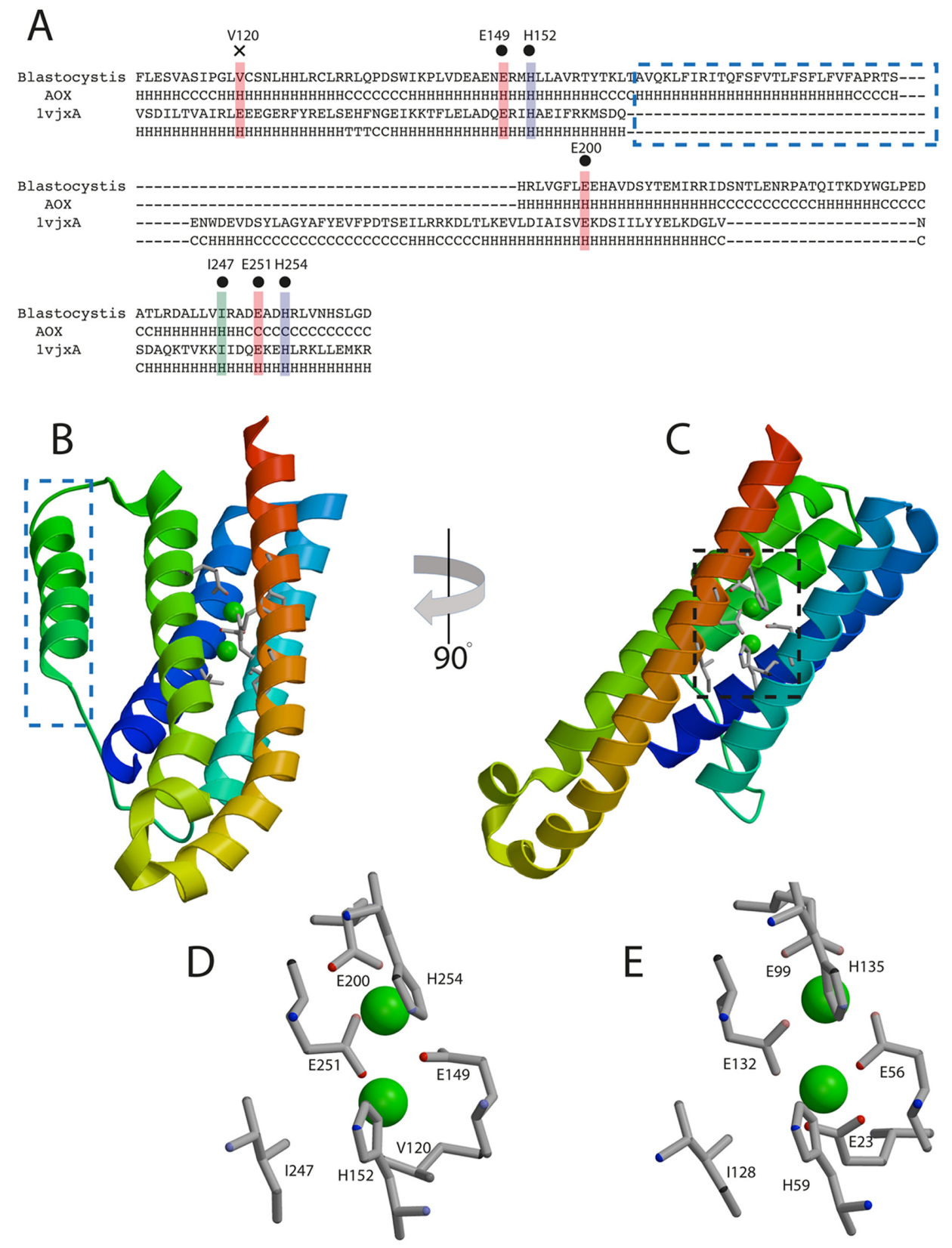

Figure 2 Structural model of Blastocystis AOX. (A) The alignment between Blastocystis AOX and a putative Ferritin-like diiron-carboxylate protein (Tm I 526) from Thermotoga maritima (PDB identifier Ivjx, chain A). Identical iron-coordinating residues are indicated by black circles and the one exception (VI20) by an ' $\mathrm{X}$ '. The predicted helix is enclosed in a dashed blue box. (B) Cartoon representation of the model with a dashed blue box indicating the inserted helix; the di-iron, taken from Desulfovibrio desulfuricans bacterioferritin (PDB identifier Infv), is represented as green spheres; iron-coordinating sidechains are represented as sticks. (C) The same model rotated $90^{\circ}$ about the vertical axis; the di-iron center is indicated by a dashed blue box. (D) The di-iron center is shown in detail with conserved residues labeled in Figure $3 \mathrm{~A}$ indicated. (E) The corresponding view of the T. maritima template (PDB identifier Ivjx) di-iron center. 
This approach has distinct advantages over constrained molecular dynamics approaches, especially when the insertion is well structured, as in the present case. ${ }^{9}$ Because the structural fragments are chosen based on predicted primary and secondary structural similarity to the query sequence, as well as tertiary structural similarity to the template at the gap end points, the resulting model contained a helix in the predicted region, while retaining the core iron-binding residues, as indicated in Figure $2 \mathrm{~B}$ and $\mathrm{C}$.

The structural model has a well-defined active site where all of the iron-coordinating residues, with the notable exception of V120, are conserved between the Blastocystis sequence and the template, as indicated in Figure 2D and E. Interestingly, the model clearly positions E200 in an ironcoordinating position, rather than E201, as predicted by the earlier $\Delta^{9}$-desaturase model. ${ }^{8}$ Given the information available at the time, the exact identity of the iron-coordinating residue at this position was ambiguous, as pointed out by the authors themselves. We investigated the issue of iron coordination further by building a model based on $\Delta^{9}$-desaturase (PDB identifier 1afr, chain A) following the same protocol described above. As shown in Table 1, the magnitude of the score for this template (5.53) was substantially lower than that of the template used here (14.1), which provides strong support for our model. Interestingly, we observed that even in our $\Delta^{9}$-desaturase model, residue E200, and not E201, was in the iron-coordinating position (results not shown). Thus, we attribute this re-assignment of the iron-coordinating residue to the alignment and modeling methods, rather than the choice of template. In conclusion, we cannot find any argument against the assignment of E200 as the iron-coordinating residue. Moreover, the assignment of E200 as an iron-coordinating ligand is supported by mutagenesis studies which showed the complete abolishment of AOX function upon mutation of the equivalent glutamate to alanine in Trypanosoma vivax; conversely, mutation of the equivalent glutamate of E201 was tolerated, albeit with reduced activity. ${ }^{15}$ Thus there are multiple lines of support for the proposed model. The FFAS03/Spanner model was supported by conservation and mutagenesis data; however, the resulting structure was by no means obvious, as evidenced by the fact that among the top-5 I-TASSER models, none had the same overall fold as the model proposed here. The recent report of crystallization of AOX from T. brucei, ${ }^{10}$ represents an interesting opportunity for a blind structure prediction that can be validated soon.

We note that residue V120 is perfectly conserved in the 100 closest homologs of Blastocystis (data not shown), but is a glutamate in the template. Most likely, this is not an alignment error, as there are no acidic residues in the immediate vicinity of the Blastocystis AOX sequence. Since a valine at this position was not found in any member of the Ferritin superfamily, it is expected that the residue in question has functional significance, such as tuning the redox potential of AOX, as has been observed in Cytochrome c6A. ${ }^{16}$ Due to its global similarity, we consider our AOX model to be a refinement of the original Andersson and Nordlund model; the improvement comes from increased structural coverage, advances in sequence alignment methods, and from use of an automated fragment assembly method.

\section{Discussion}

The proton gradient across the mitochondrial inner membrane generated by the electron transport chain and oxidative phosphorylation is an extremely powerful driver of cellular ATP production. ${ }^{17}$ Four membrane complexes (Complex I or NADH:quinone oxidoreductase, Complex II or succinate dehydrogenase, Complex III or the cytochrome bc complex $_{1}$ and Complex IV or cytochrome c oxidase) work in conjunction to generate an electrochemical gradient by pumping protons across the mitochondrial inner membrane. Complex IV couples this electron transport to the reduction of oxygen to water. The resulting proton gradient is subsequently harvested via the $\mathrm{F}_{0} \mathrm{~F}_{1}$ ATP synthase (sometimes called Complex V) to produce ATP. ${ }^{17}$

However, eukaryotes living in oxygen-poor or even anoxic environments cannot use oxygen as the terminal electron acceptor and must use other, organic (eg, example fumarate) or inorganic ( such as $\mathrm{NO}_{2}^{-}$or $\mathrm{NO}_{3}^{-}$), terminal acceptors and subsequently have shorter mitochondrial electron transport chains. ${ }^{18}$ Several eukaryotes living in these environments have, in addition, unusual mitochondria that are devoid of many features considered typically mitochondrial, such as cristae, an organellar genome, citric acid cycle and, as mentioned above, an electron transport chain. ${ }^{19}$ The most extreme mitochondria are the mitosomes, as found in microsporidia, Giardia, and Entamoeba, and whose functions are not quite clear but considering their abundance must be important for these organisms. ${ }^{19}$ Another class of unusual mitochondria are the hydrogenosomes that produce molecular hydrogen as the end product of a short electron chain with protons as terminal electron acceptors. ${ }^{19}$ Recently, organelles with more intermediate features of both classic mitochondria and hydrogenosomes have been discovered in the distantly related stramenopile Blastocystis ${ }^{5}$ and ciliate Nyctotherus ovalis. ${ }^{20}$ These organelles do contain 
Complex I and Complex II but do not have any of the other electron transport complexes (although $N$. ovalis seems to contain a cytochrome $\mathrm{c}_{1}$ homolog, it however contains a premature stop-codon). ${ }^{20}$

A novel approach to selectively fight parasites such as Blastocystis $^{5}$ and trypanosomes ${ }^{3,4}$ is to target key metabolic enzymes such as AOX that are not present in humans. Structure-based drug design relies on high-quality modeling, especially in the active site region. However, the closest homologs to Blastocystis AOX are similar only at the superfamily level. Structural modeling in cases of very remote similarity is problematic for at least two reasons. First, it is generally difficult to assign a high degree of confidence in the accuracy of the resulting alignment. Second, an accurate alignment will often contain many gaps that are difficult to model using conventional tools. AOX presents an interesting exception to the first problem, as much is known about functionally important and/or conserved residues. However, the existence of a nearly 30-residue gap nevertheless presents a significant modeling challenge. The program Spanner was developed to automatically address problems such as this where distant homolog structure can be used as a scaffold on which structural fragments can then be grafted in gap regions to generate a hybrid template that has no gaps with respect to the original query. This type of hybrid template alignment can also be performed by hand, but is very labor intensive, and it is difficult to balance the various constraints (primary, secondary, and tertiary) required to generate a consistent structure.

The residues predicted to bind the di-iron complex in the Spanner model agree in general with a previously published model generated by hand. ${ }^{8}$ However, one key difference is that E200 is placed in an iron-coordinating position, rather than E201, as assigned by the earlier model. Given this small but unambiguous difference, along with the placement of the inserted helix, the AOX model represents an ideal test case for the proposed modeling methodology. As discussed above, mutagenesis experiments support the assignment of E200 to the iron ligating position. The fact that crystallization of AOX from $T$. brucei has recently been reported ${ }^{10}$ suggests that we can expect to validate our blind prediction in the near future.

Finally, because the active site region appears to match the template extremely well, it is expected to provide further insight into AOX function. This finding represents a natural extension of the present project toward structure-based drug design. Clearly, a crystal structure of AOX will provide more valuable information for structure-based drug design than a model built on a remote homolog, as presented here.
Moreover, because our model is globally similar to the earlier model built on a different family member from the same superfamily, we cannot claim that it provides deep new insights into the function of AOX, other than the reassignment of E200 as the iron-coordinating residue in agreement with mutagenesis studies. ${ }^{15}$ The use of structural genomics targets in structure-based drug design depends on optimal use of sequence and structural information. Demonstrating an automated hybrid structural model assembly in a blind prediction, which can quickly be validated is, thus, of great value to the structural biology community.

\section{Materials and methods Modeling}

A structural model of Blastocystis AOX was constructed as follows. First, the full amino acid sequence was submitted to the BioInfoBank MetaServer, ${ }^{7}$ from which a consensus assignment to the Ferritin-like SCOP superfamily was made for the C-terminal 203 residues (N102-A304). This subsequence was then re-submitted to the FFAS03 server. ${ }^{11}$ The top-scoring alignment was to a putative Ferritin-like diironcarboxylate protein (Tm1526) from Thermotoga maritima, (PDB identifier 1vjx, chain A). The sequence identity to 1vjxA was only $11 \%$; however, with the exception of V120, all the iron-coordinating residues were conserved. In this alignment, a predicted helix (A164-S192) was aligned with a long loop in the template. The alignment was thus modified by hand so that this predicted helix-containing region was aligned with a large gap, as shown in Figure 2A. The modified alignment was then submitted to the Spanner server at http://sysimm.ifrec.osaka-u.ac.jp/spanner/, which has been described in detail previously. ${ }^{9}$ In brief, Spanner uses structural fragments to generate a hybrid template such that there are no gaps with the query. It accomplishes this by querying a database of structural fragments using the geometry of the endpoints of the gaps, along with the corresponding query sequence and secondary structure as input. Many fragments are sampled and the one having the closest overall match to the input is retained. In the case of AOX, the main use of Spanner was to automatically search known structures for a fragment that was similar at the endpoints to the long loop in the template 1vjxA, but contained a helix. Since many candidate fragments exist, the final selection is made based on a number of factors, including sequence similarity to the query, and absence of clashes with the rest of the template. In order to illustrate the fragment assembly approach used by Spanner, three representative fragments (of many thousands sampled) are shown in Figure 3. After fragment selection and 


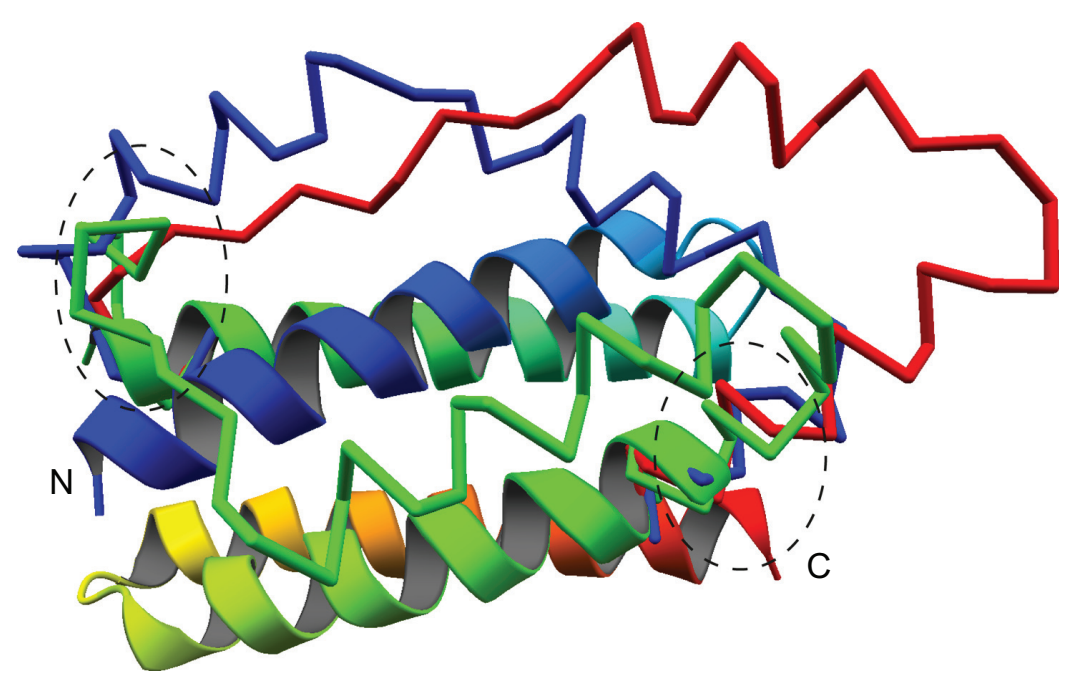

Figure 3 Spanner fragment assembly. Three representative fragments each of length 32 residues are shown in backbone representation in blue, green, and red with the blue fragment the highest-scoring. The template is shown in cartoon representation with rainbow coloring; the $\mathrm{N}$ (blue) and $\mathrm{C}$ (red) helix termini are indicated. The anchor points, used as restraints in fragment selection, are enclosed in dashed ovals. Each of the three fragments is shown with anchor residues superimposed on those of the template.

assembly, sidechains were replaced and the all-atom model was refined by restrained energy minimization. Note that in order to highlight the iron-coordinating residues, the iron atoms were transferred from PDB entry $1 \mathrm{nfv}$ (Desulfovibrio desulfuricans bacterioferritin) to the template $1 \mathrm{vjx}$ and to the Spanner model. This was achieved by structurally superimposing the $1 \mathrm{nfv}$ structure onto $1 \mathrm{vjx}$ and extracting the iron atoms from the superimposed structure. As a reference, 3D models were also constructed using the I-TASSER online server (http://zhanglab.ccmb.med.umich.edu/I-TASSER/).

\section{Phylogenetic analysis}

The conceptually translated Blastocystis alternative oxidase was aligned using Clustal $\mathrm{W}^{21}$ as implemented in BioEdit ${ }^{22}$ to reference sequences from Genbank after organellar targeting sequences and the 28 residue helical insertion of AOX was removed. The alignments were manually refined and only unambiguously aligned regions were used for phylogenetic analysis, leaving a data set of 12 taxa with 142 amino acid positions which were subsequently analyzed by Bayesian and maximum-likelihood (ML) corrected distance methods (see details below). Likelihood searches were performed in a Bayesian framework under the mixed amino acid substitution model accommodating site rate variation (fraction of invariable sites plus four variable gamma rates) using the program MrBayes ${ }^{23}$ as implemented by the University of Oslo Bioportal (freely available at www.bioportal.uio. no). All analyses started with randomly generated trees and ran for 500,000 generations, with sampling at intervals of 1000 generations that produced 500 trees. The log-likelihood values of the 500 trees in each analysis were plotted against the generation time (not shown). All trees produced prior to reaching stationarity were discarded, ensuring that burn-in samples were not retained. Although the likelihood model stabilized very rapidly, only the last 400 trees of analysis were used to estimate separate $50 \%$ majority rule consensus trees for these. The frequency of any particular clade, among the individual trees contributing to the consensus tree, represents the posterior probability of that clade. ${ }^{23}$ For the maximum likelihood analyses, protein data sets were analyzed using PHYML ${ }^{24}$ under the WAG model with a mixed four-category discrete gamma plus invariable sites model of rate heterogeneity using 100 bootstraps.

\section{Acknowledgments}

The authors would like to thank the developers of Spanner, in particular Mieszko Lis (MIT) and Jamica Joy Sarmiento (Osaka University), for technical help. MvdG is grateful for support of the Wellcome Trust (078566/A/05/Z). DMS was partially supported by the Japan Society for the Promotion of Science (JSPS) through its "Funding Program for World-Leading Innovative R\&D on Science and Technology (FIRST Program).

\section{Disclosure}

The authors have no conflicts of interest to declare.

\section{References}

1. Zierdt CH. Blastocystis hominis - past and future. Clin Microbiol Rev. 1991;4(1):61-79.

2. Stenzel DJ, Boreham PF. Blastocystis hominis revisited. Clin Microbiol Rev. 1996;9(4):563-584. 
3. Nakamura K, Fujioka S, Fukumoto S, et al. Trypanosome alternative oxidase, a potential therapeutic target for sleeping sickness, is conserved among Trypanosoma brucei subspecies. Parasitology International. 2010;59(4):560-564.

4. Nihei C, Fukai Y, Kita K. Trypanosome alternative oxidase as a target of chemotherapy. Biochimica et Biophysica Acta (BBA) - Molecular Basis of Disease. 2002;1587(2-3):234-239.

5. Stechmann A, Hamblin K, Pérez-Brocal V, et al. Organelles in Blastocystis that blur the distinction between mitochondria and hydrogenosomes. Curr Biol. 2008;18(8):580-585.

6. Denoeud F, Roussel M, Noel B, et al. Genome sequence of the stramenopile Blastocystis, a human anaerobic parasite. Genome Biol. 2011;12(3):R29.

7. Ginalski K, Elofsson A, Fischer D, Rychlewski L. 3D-Jury: a simple approach to improve protein structure predictions. Bioinformatics. 2003; 19(8):1015-1018.

8. Andersson ME, Nordlund P. A revised model of the active site of alternative oxidase. FEBS Lett. 1999;449(1):17-22.

9. Lis M, Kim T, Sarmiento J, et al. Bridging the gap between singletemplate and fragment based protein structure modeling using Spanner. Immunome Res. 2011;7(1):1-8.

10. Kido Y, Shiba T, Inaoka DK, et al. Crystallization and preliminary crystallographic analysis of cyanide-insensitive alternative oxidase from Trypanosoma brucei brucei. Acta Crystallogr Sect F Struct Biol Cryst Commun. 2010;66(Pt 3):275-278.

11. Jaroszewski L, Rychlewski L, Li Z, Li W, Godzik A. FFAS03: a server for profile-profile sequence alignments. Nucleic Acids Res. 2005;33(Web Server issue):W284-W288.

12. Gomes CM, Le Gall J, Xavier AV, Teixeira M. Could a diironcontaining four-helix-bundle protein have been a primitive oxygen reductase? ChemBioChem. 2001;2(7-8):583-587.

13. Andrews SC. The Ferritin-like superfamily: Evolution of the biological iron storeman from a rubrerythrin-like ancestor. Biochim Biophys Acta. 2010;1800(8):691-705.
14. Lane N. Life Ascending: The Ten Great Inventions of Evolution. London: Profile Books Ltd; 2009.

15. Nakamura K, Sakamoto K, Kido Y, et al. Mutational analysis of the Trypanosoma vivax alternative oxidase: the $\mathrm{E}(\mathrm{X}) 6 \mathrm{Y}$ motif is conserved in both mitochondrial alternative oxidase and plastid terminal oxidase and is indispensable for enzyme activity. Biochem Biophys Res Commun. 2005;334(2):593-600.

16. Worrall JA, Luisi BF, Schlarb-Ridley BG, Bendall DS, Howe CJ. Cytochrome c6A: discovery, structure and properties responsible for its low haem redox potential. Biochem Soc Trans. 2008;36(Pt 6): 1175-1179.

17. Scheffler IE. Mitochondria. 2nd ed. Hoboken, New Jersey: John Wiley and Sons, Inc; 2008.

18. Tielens AGM, Rotte C, van Hellemond JJ, Martin W. Mitochondria as we don't know them. Trends Biochem Sci. 2002;27(11):564-572.

19. van der Giezen M. Hydrogenosomes and mitosomes: conservation and evolution of functions. J Euk Microbiol. 2009;56:221-231.

20. Boxma B, de Graaf RM, van der Staay GW, et al. An anaerobic mitochondrion that produces hydrogen. Nature. 2005;434(7029):74-79.

21. Thompson JD, Higgins DG, Gibson TJ. CLUSTAL W: Improving the sensitivity of progressive multiple alignment through sequence weighting, positions-specific gap penalties and weight matrix choice. Nucl Acids Res. 1994;22(22):4673-4680.

22. Hall TA. BioEdit: a user-friendly biological sequence alignment editor and analysis program for Windows 95/98/NT. Nucl Acids Symp Ser. 1999;41:95-98.

23. Ronquist F, Huelsenbeck JP. MrBayes 3: Bayesian phylogenetic inference under mixed models. Bioinformatics. 2003;19(12):1572-1574.

24. Guindon S, Dufayard JF, Lefort V, Anisimova M, Hordijk W, Gascuel O. New algorithms and methods to estimate maximumlikelihood phylogenies: assessing the performance of PhyML 3.0. Syst Biol. 2010;59(3):307-321.
Research and Reports in Biochemistry

\section{Publish your work in this journal}

Research and Reports in Biochemistry is an international, peer-reviewed, open access journal publishing original research, reports, reviews and commentaries on all areas of biochemistry. The manuscript management system is completely online and includes a very quick and fair

\section{Dovepress}

peer-review system. Visit http://www.dovepress.com/testimonials.php to read real quotes from published authors. 